\title{
Energy Performance in Italian Public Administration
}

\author{
Daniela Sica, Ornella Malandrino and Benedetta Esposito \\ Department of Business Sciences-Management \& Innovation Systems (DISA-MIS), University of Salerno, Fisciano (SA) 84084, Italy
}

\begin{abstract}
The commitment to improve energy and environmental performance in public administration (PA) is essential for the success of development models geared towards lasting sustainability. The PA has a dual consumer/user role, public heritage and decision maker/planner, in promoting energy efficiency at local level, in the light of specific problems and peculiarities. For several decades, initiatives have been launched at various levels to improve energy and environmental performance in the PA. The aim of this study is to outline from a critical perspective, the state of the art of policy makers in Italy concerning energy efficiency measures in PA and the main issues impeding the achievement of its full potential. We also analyze the driving forces for implementing systems and tools to improve energy efficiency. The European Union Directive (2012/27/EU) recently adopted in Italy gives the PA the task of promoting energy efficiency at national level by defining and implementing policies and measures aimed at improving energy performance.
\end{abstract}

Key words: Energy performance, PA, efficiency.

\section{Introduction}

For several decades, a plethora of initiatives have been undertaken at various levels to improve the energy and environmental performance of the public administration (PA), shifting paradigms from the current socio-economic development perspective, to review them from the "sustainable" development point of view.

Since the United Nations Conference on Environment and Development, held in Rio de Janeiro in 1992, useful operational tools have been introduced and used by local authorities in order to ensure effective and efficient land management, based on the fundamental concept of "accountability”. Accountability is concerned not only with reporting non-financial performance, but also a set of promotional and information activities, through transparent decision-making processes [1].

Since the 1990s, the Italian organism PA, consuming more than 1,000 toe, has been obliged to appoint an Energy Manager (EM) to promote and

Corresponding author: Daniela Sica, Ph.D., research fields: circular economy, sustainable management, energy systems and corporate social responsibility. spread a culture of energy efficiency within the organization.

However, in spite of regulatory constraints, to date the Italian PA has appointed very few EMs and remains distinguished by much inefficiency.

In 2017, about 2,350 EMs were appointed, of whom only 180 were within the PA. While it is impossible for us to make a more accurate estimate, the regulation appears to have been widely disregarded, since only half of the metropolitan cities have sent the nomination, the metropolitan cities of Cagliari, Messina, Palermo, Bari, Florence, Bologna and Genoa are absent. The provincial capitals that have appointed an EM are 34 out of 116 . There are 57 non-capital municipalities, compared to 1,094 in total, which have more than 10,000 inhabitants. The nomination rate for the regions is very low and similar to that of the provinces, 6 out of 20 for the first 23 out of 93 for the latter. Finally, as regards the Ministries, it is not easy to evaluate the rate of non-fulfillment of the appointment for the various Italian Ministries due to the complex organizational chart that characterizes them. Certainly at least one EM for each Ministry would be desirable considering that in 2017 only 2 appointments of EMs were received by the Ministry 
of the Interior [2].

Therefore, it is a well-known fact that the PA's commitment to improve energy performance is long overdue even if recent studies have estimated that by 2020 the PA could save electricity and thermal energy equal to 0.8 TWh and 1.5 TWh respectively through intervention capable of generating substantial investments valued at approximately $€ 1,000$ billion per year [3].

\section{Towards Improving Energy Efficiency in the Italian PA}

The driving forces for implementing systems and tools to improve energy efficiency range from economic and organizational oriented incentives to those related to environmental and social dimensions. The high cost of energy makes it essential to identify rapidly the most appropriate technological and organizational interventions for reducing the energy costs of the PA and its exposure to volatile energy prices [4].

In order to improve energy performance, the energy management practices of the PA must be in synchrony with the concept of energy efficiency, effective detection systems have to be implemented and suitable data and information must be available for evaluating the results obtained in order to identify possible areas of improvement.

There are however many cultural, value-related, financial, technological, institutional and operational issues in the PA sector that could limit investments in energy efficiency. It is particularly important to note that if on one hand the PA shows a lack of knowledge and unwillingness to adopt practices and systemic tools for monitoring and control, on the other, it appears to be too complicated and costly. In fact, specific skills and high technical and organizational capabilities are required when implementing programs aimed at efficient and sustainable energy management.

There are also other problems such as the persisting limited availability of financial resources, which discourages the PA from making plans and investments that could yield returns in the medium-long term, which calls for the realization of effective energy efficiency improvement strategies.

In addition, the lack of awareness of the benefits and opportunities related to the improvement of energy performance does not permit the development of a "culture" of shared energy efficiency.

Culture in this case expressed both in “socio-environmental” terms (e.g. environmental protection, enhancement of energy and renewable resources, etc.) and in "individualistic" terms (e.g. everyday use energy conscious, lifestyle, buying patterns, etc.) depends on the degree of sharing of objectives, strategies and values that the PA can spread internally and among the various external stakeholders. Internally, through the virtuous cycle of strategic sharing and co-responsible design, using communication and training to "contaminate" vertically and across the organization and stimulate feedback flows; externally, through the enhancement in a communicational key, of what has been achieved and the involvement of all stakeholders, whatever their title of relationship with the organization.

From a technical and operational perspective, the complexity of the planning, technical and financial aspects of energy efficiency measures, as well as the lack of commitment, also relational, of the individuals responsible for their implementation, magnify and reinforce the barriers to energy efficiency.

Additionally legislative instability and the long and tortuous bureaucratic procedures required by global operations are factors that induce the PA to opt for simple technologies to implement rapidly but characterized by short-term vision.

One way of overcoming knowledge barriers and reducing technical and economic information asymmetries, may be to enforce the regulations currently in force.

\section{Conclusion}


The affirmation of energy paradigms geared towards greater efficiency and sustainability is an interesting challenge and presents new opportunities for the Italian PA, which should play a leading role in the complex economic scenario that is emerging for some years [5].

The achievement of the multiple benefits linked to the implementation of interventions aimed at improving energy performance requires, on the part of the PA, a rethinking of organizational and functional models, looking for more flexible and less bureaucratic forms of management, and a more dynamic, pervasive approach and proactive towards initiatives that promote energy efficiency.

The current energy efficiency approach of the Italian PA stems from a strategically unstructured vision, governed by legislation and highly fragmented planning that result in occasional activities rather than medium-long term planned initiatives, enacted after a careful measurement and assessment of the results obtained.

Although the adoption of dimension tailored interventions and, more in general, the diffusion of a solid culture of energy efficiency highlights, as we have seen, numerous problems and barriers, it is essential to move towards new governance systems. The latter, borrowing managerial logics, must allow the formulation of structured energy plans on the results-objective of management, the preparation of an operational program and the systematic verification of the energy performance achieved with the objectives set, with the consequent provision of corrective actions led by the feedback.

There are, in fact, ample margins for the improvement of energy efficiency in the Italian PA, both in relation to the internal dimension-primarily in the management of human resources, in terms of training information and awareness, making these issues more familiar and highlighting their potential and the repercussions on the overall performance of the organization-both in relation to the external dimension, through for example the involvement of all those who interact with the PA, such as suppliers, local communities, energy service companies, organizations and market operators for energy efficiency.

Therefore, it appears that the boundaries of the PA require redefining in order to reconsider the relationships between the public and private sectors and the public institutions in terms of cooperation, collaboration, and partnership, based on reliable and long-lasting relationships, integration of public funds and private resources and the resultant risk allocation. Moreover, it is essential to exchange experiences and best practices with the aim of guiding the PA and especially local bodies towards lasting sustainability and economic growth through competitiveness, environmental protection, and social development.

Finally, it is necessary to highlight that governance for sustainability requires a well-functioning PA with effective organizations and highly qualified, committed staff. Effective organizations have the capacity to deliver results while making change happen. They are able to successfully tackle issues such as performance, risk management, uncertainty, partnerships, assets, human capital, etc.

\section{References}

[1] CONSIGLIO NAZIONALE DEI DOTTORI COMMERCIALISTI E DEGLI ESPERTI CONTABILI-CNDCEC. 2011. "Contabilità e rendicontazione di sostenibilità del sistema della pubblica amministrazione e degli enti locali.” Policy Statement. Accessed Dec. 2016. http://www.cndcec.it/Portal/Default.aspx.

[2] FIRE. 2018. "Rapporto 2018 sugli Energy Manager in Italia: Indagini, Evoluzione del ruolo e Statistiche.” Accessed October 21, 2018 http://fire-italia.org/wp-content/uploads/2018/07/2018-07-ra pporto-energy-manager-2018.pdf.

[3] POLITECNICO DI MILANO. 2013. L'efficienza energetica in Italia: soluzioni tecnologiche ed opportunità di business nell'industria, i servizi e la Pubblica Amministrazione. Energy efficiency report. Accessed Dec. 05, 2015. http://www.energystrategy.it/ 
[4] De Paoli, L., and Bongiolatti, L. 2006. "The Promotion of Energy Efficiency in Italy.” Economics and Policy of Energy And The Environment 3: 29-68.
[5] Borgonovi, E. 2005. Principi e sistemi aziendali per le amministrazioni pubbliche. Egea, Milano. 